\title{
Compléments à un théorème de T. S. Broderick concernant les événements dépendants
}

\author{
Par Maurice Fréchet.
}

(Received 28th June, 1939. Read 3rd November, 1939.)

Introduction. Nous nous proposons ici de pousser un peu plus loin les conséquences que Broderick a tirées d'un théorème dont il nous a fait connaître l'existencel au dernier Colloque de St Andrews.

L'un des buts de Broderick était de prouver que certaines formules de King restent valables quand les événements considérés ne sont plus indépendants, démonstration dont il n'avait pas trouvé trace dans la littérature, sauf relativement à des cas particuliers.

Nous avons appris depuis et nous faisons ici connaître que de telles démonstrations ont été publiées en 1934 par Charles Jordan ${ }^{2}$ et en 1936 par Carlo Bonferroni ${ }^{3}$.

La méthode suivie par Charles Jordan utilise d'ajlleurs implicitement un théorème identique à celui de Broderick, sans que ce théorème soit explicitement énoncé, ni démontré.

Ces auteurs, ayant à calculer des probabilités d'événements déterminés quand on se donne les événements $A_{1}, \ldots A_{m}$, font intervenir essentiellement les événements

$$
I_{a_{1}} \ldots a_{r} \bar{a}_{r+1} \ldots \bar{a}_{m} \equiv A_{a_{1}} \ldots A_{a_{r}} \bar{A}_{a_{r+1}} \ldots \bar{A}_{a_{m}}
$$

consistant dans le concours de $A_{a_{1}}, \ldots, A_{a_{r}}$ et des "événements complémentaries" des autres $A_{j}$.

On comprend mieux la raison et le succès de cette méthode quand on se reporte à l'un des résultats d'un mémoire que nous avions publié en 1923 sur la théorie des ensembles. Transporté, suivant le procédé classique, dans la théorie des événements, ce résultat

${ }^{1}$ On some symbolic formulae in probability theory, by T. S. Broderick, Proc. Royal Irish Academy, 44 (1937), 19-28.

" Le théorème de probabilité de Poincaré généralisé au cas de plusieurs variables indépendantes, Actr Scient. Math., Szeged, 7 (1934), 106.

3 Teoria statistica della classi e calcolo delle probabilita, R. Instituta Sc. Econom. $e$ Conm., Firenze, 1936.

4 Des familles et fonctions additives d'ensembles abstraits, Fund. Mathem., 4 (1923), $333-365$, et 5 (1924), 206-251. 
particulier se traduit de la façon suivante: en définissant convenablement l'additivité d'une famille d'événements, la famille des $2^{m}$ événements $I_{a_{1}} \ldots a_{r} \vec{a}_{r+1} \ldots \vec{a}_{m}$ constitue "la plus petite" famille additive d'événements comprenant les événements $A_{1}, \ldots A_{m}$. C'est pourquoi nous avions appelé ces événements $I$, les "atomes" du système $A_{1}, \ldots A_{m}$. Cependant, pour faciliter la lecture de ce qui suit. nous ne ferons usage ici, ni de ce résultat, ni d'aucun résultat de ce mémoire de 1923.

Les événements fonctions d'événements. Il est naturel de dire $q u$ 'un événement $F$ est fonction des événements $A_{1}, \ldots A_{m}$, quand $F, A_{1}, \ldots A_{m}$ étant definis sur la même catégorie d'épreuves, il n'est pas nécessaire de connaître le résultat complet d'une épreuve pour savoir si $F$ s'est produit ou non, il suffit de savoir quels sont, dans cette épreuve, ceux des $A_{j}$ qui ont eu lieu et ceux des $A_{j}$ qui n'ont pas eu lieu.

Il en résulte que toute fonction $F$ des événements $A_{1}, \ldots A_{m}$ est un événement qui consiste dans la réalisation de l'un quelconque des événements $I_{a_{1}} \ldots a_{r} \bar{a}_{r+1} \ldots \bar{a}_{m}$ qui appartiennent à un groupement $G$ de certains de ces $I$. Les événements $I$ sont d'ailleurs incompatibles; on peut dire que $F$ est la somme des $I$ d'un certain groupement $G$ extrait de l'ensemble des $I$ :

$$
F=\sum_{G} I_{a_{1}} \ldots a_{r} \bar{a}_{r+1} \ldots \bar{a}_{m} .
$$

Ceci étant, il y a avantage à faire apparaître dans la démonstration de Broderick deux parties très distinctes qui s'y trouvent confondues: $1^{0}$ démonstration d'un lemme sur les événements dont l'énoncé a été partiellement donné par Charles Jordan: $2^{0}$ déduction, alors immédiate, du théorème, en faisant intervenir les probabilités de ces événements. Cette façon de faire a l'avantage de faire ressortir un lemme utile et d'en permettre l'application à des généralisations et des compléments.

Appelons événements $B$, des événements de la forme

$$
B=A_{j_{1}} \ldots A_{j_{r}} \bar{A}_{j_{r+1}} \ldots \bar{A}_{j_{r+4}}
$$

où $j_{1} \ldots j_{r+s}$ est une combinaison de $m$ ou de moins de $m$ des $m$ premiers entiers. Les $I$ figurent (pour $r+s=m$ ) parmi les $B$. On appellera événements $B$ négatifs ceux dont le symbole comporte au moins un signe moins le nombre de ces signes moins étant l'ordre de cet événement négatif. On appellera événements $B$ positifs les autres $B$. 
Lemme. Tout événement $F$ fonction des événements $A_{1} \ldots A_{m}$ peut être obtenu par un nombre fini d'additions et de soustractions à partir d'événements $B$ positifs, les additions portant sur des événements incompatibles, les soustractions ne retranchant d'un événement $D$ qu'un événement $C$ impliquant $D$.

Il suffit évidemment de prouver ce lemme quand $F$ se réduit à un des événements $I$. C'est ici que nous pouvons utiliser le raisonnement de Broderick mais sans y faire intervenir comme lui les probabilités.

On peut écrire d'après $(3)$ :

$$
B+A_{j_{1}} \ldots A_{j_{r}} A_{j_{r+1}} \bar{A}_{j_{r+2}} \ldots \bar{A}_{j_{m}}=A_{j_{1}} \ldots A_{j_{r}} \bar{A}_{j_{r+2}} \ldots \bar{A}_{j_{m}}
$$

ou $B+B^{\prime}=B^{\prime \prime}$ ou $B=B^{\prime \prime}-B^{\prime}$. Ici $B^{\prime}$ implique $B^{\prime \prime}$, et $B^{\prime \prime}$ et $B^{\prime}$ restent du type $B$ mais avec un nombre de signes moins inférieur à celui qui correspond à $B$.

On pourra alors appliquer cette méthode à un des $I$, qui deviendra $B_{1}^{\prime}-B_{1}$, puis on l'appliquera à $B_{1}^{\prime}$ et $B_{1}$ et ainsi de suite. Les ordres des $B$ négatifs diminuant à chaque fois finiront par devenir nuls au bout d'un nombre fini d'opérations, comme il a été annoncé.

Remarque.-Jordan donne même une règle pour effectuer cette réduction: on remplace chaque $\bar{A}_{j}$ par $E-A_{j}, I$ devient

$$
A_{a_{1}} \ldots A_{a_{r}}\left(E-A_{a_{r+1}}\right) \ldots\left(E-A_{a_{n}}\right),
$$

on effectue les multiplications comme s'il s'agissait d'un produit algébrique, puis on remplace dans le résultat obtenu $E^{\gamma}$ par $E$ et $E^{\gamma} A_{i}$ par $A_{i}$. Par exemple

$$
\left(E-A_{1}\right)\left(E-A_{2}\right)=E-A_{1}-A_{2}+A_{1} A_{2}
$$

mais il ne donne pas de démonstration de cette règle et ne dit pas non plus quel sens il faudra attribuer au second membre. Si par exemple $E-A_{1}$ et $E-A_{2}$ sont incompatibles, $A_{2}$ a lieu nécessairement quand $E-A_{1}$ a lieu. Il est donc naturel de considérer $E-A_{1}-A_{2}$ comme l'impossibilité et par suite $E-A_{1}-A_{2}+A_{1} A_{2}$ comme désignant $A_{1} A_{2}$, alors que $\left(E-A_{1}\right)\left(E-A_{2}\right)$ est par hypothèse impossible.

Au contraire, la méthode de Broderick démontre complètement le lemme que nous avons énoncé.

Théorème de Broderick.-Ce théorème s'obtient alors immédiatement en appliquant le théorème des probabilités totales à chacune des opérations qui conduisent à la démonstration du lemme. On en 
conclut avec Broderick, en termes différents mais équivalents: la probabilité d'un événement $H$ fonction de $m$ événements $A_{1}, \ldots A_{m}$ peut s'exprimer sous la forme

$$
\text { Prob. } H=\sum_{r=0}^{m} \sum_{\beta_{1} \ldots \beta_{r}}^{\Sigma} c_{\beta_{1} \ldots \beta_{r}} \text { Prob. }\left\{A_{\beta_{1} \ldots .} A_{\beta_{r}}\right\}
$$

où les $c_{\beta_{1}} \ldots \beta_{r}$ sont des nombres entiers $(>0,<0$ ou $=0$ ) et où le terme correspondant à $r=0$ peut s'écrire $c_{0} \operatorname{Pr} E$ ou simplement $c_{0}, c_{0}$ étant d'ailleurs égal à 1 ou 0 suivant que $\overline{A_{1}} \ldots \overline{A_{m}}$ implique $H$ ou non.

Il y a un complément essentiel à apporter à l'énoncé de Broderick et qui d'ailleurs résulte immédiatement de sa démonstration, c'est que sa méthode fournit pour les $c$ des valeurs qui ne dépendent que de la fonction $F$ des $A_{j}$-qui sont indépendantes de la nature des $A_{j}$ et en particulier de leurs probabilités (simples ou composées).

Broderick remarque d'ailleurs que son théorème subsiste si l'on remplace les probabilités par les fréquences des $A$ ou (si les $A$ sont des ensembles), par leurs mesures. On peut porter plus loin cette remarque en disant que le théorème subsiste si l'on remplace les probabilités par une fonction additive (non nécessairement positive) d'événement. (On dira que $F(G)$ est une fonction additive de l'événement $G$ si $G_{1}$ et $G_{2}$ étant deux événements incompatibles on a

$$
F\left(G_{1}+G_{2}\right)=F\left(G_{1}\right)+F\left(G_{2}\right) \text {.) }
$$

Théorème 1. Soit $H$ un événement fonction des événements $A_{1}, \ldots A_{m}$ et $F(G)$ une fonction additive d'événement; on a alors

$$
F(H)=\sum_{r=0 \beta_{1} \ldots \beta_{r}}^{m} c_{\beta_{1} \ldots \beta_{r}} F\left(A_{\beta_{1} \ldots} \ldots A_{\beta_{r}}\right)
$$

où le terme correspondant à $r=0$ est $c_{0} F(E)$ et où les $c_{\beta_{1}} \ldots \beta_{r}$ sont des entiers $(>0,<0$ on $=0)$ indépendants à la fois de la fonction $F$ et de la nature des événements $A_{1}, \ldots A_{m}$. Et cette expression est unique en ce sens qu'il est impossible d'y remplacer les entiers $c$ par d'autres nombres (entiers ou non) jouissant des mêmes propriétés.

C'est cette unicité qui reste seulement à démontrer. A cet effet, supposons qu'il existe une expression analogue de $F(H)$ où les coefficients $c$ sont remplacés par d'autres coefficients $c^{\prime}$. En appelant $c^{\prime \prime}$ les différences $c-c^{\prime}$, on aurait

$$
\sum_{r=0 \beta_{1} \ldots \beta_{r}}^{m} c_{\beta_{1} \ldots \beta_{r}}^{\prime \prime} F\left(A_{\beta_{1} \ldots} A_{\beta_{r}}\right)=0 .
$$

Puisque les $c^{\prime \prime}$ (comme les $c$ et les $c^{\prime}$ ) sont indépendants de $F$ et de la 
nature des $A$, prenons pour les $A$ des événements indépendants et pour $F(G)$ la probabilité de $G$. En posant $p_{i}=$ Prob. $A_{i}$, on aura:

$$
\sum_{r=0}^{m} \sum_{\beta_{1}}^{m} c_{\beta_{r}} c_{\beta_{1} \ldots \beta_{r}}^{\prime \prime} p_{\beta_{1}} \cdots p_{\beta_{r}}=0
$$

Le premier membre est un polynome en $p_{1}, \ldots p_{m}$ du premier degré par rapport à chaque $p_{i}$ et il doit être nul quelles que soient les valeurs des $p_{i}\left(\geqq 0\right.$ et $\leqq 1$ ). Donc les coefficients $c^{\prime \prime}{ }_{\beta_{i}} \ldots \beta_{r}$ sont nuls comme il fallait le prouver.

C'est précisément le mode de raisonnement employé par Broderick pour appliquer son théorème à la démonstration des formules de King. On voit que ce raisonnement peut aussi fournir un résultat beaucoup plus général.

On en tire aussi une règle souvent très commode.

Il arrive en effet souvent qu'on trouve immédiatement ou très facilement la probabilité d'un événement $H$ fonction des $A$ quand on sait (ou l'on suppose) que ces $A$ sont indépendants. On passera alors immédiatement au cas de la dépendance par la règle suivante.

Règle. Si l'on sait calculer la valeur de la probabilité d'un événement $H$ fonction de plusieurs événements $A_{1}, \ldots A_{m}$ dans le cas où les $A$ sont indépendants, sous une forme valable quelles que soient les probabilités $p_{i}=$ Prob. $A_{i}$, on obtient une expression de Prob. $H$ valable sans supposer les $A$ indépendants en remplaçant dans la première expression les produits $p_{\beta_{1}} \ldots p_{\beta_{r}}$ par les probabilités

$$
p_{\beta_{1}} \ldots \beta_{r}=\text { Prob. }\left\{A_{\beta_{1} \ldots} A_{\beta_{r}}\right\} \text {. }
$$

On rejoint de cette façon le symbolisme de Jordan (mais en évitant les objections formulées ci-dessus), par exemple, en écrivant symboliquement

$$
\begin{aligned}
\operatorname{Pr} .\left\{A_{a_{1}} \ldots A_{a_{r}}\left(E-A_{a_{r+1}}\right) \ldots .\right. & \left.\left(E-A_{a_{m}}\right)\right\} \\
& =\left(\left(p_{a_{1} \ldots .} p_{a_{r}}\left(1-p_{a_{r+1}}\right) \ldots\left(1-p_{a_{i,}}\right)\right)\right) .
\end{aligned}
$$

Pour effectuer l'opération symbolique du second membre, on développe le produit des $p$ et des $(1-p)$ comme si c'était un produit algébrique et, dans le résultat, on remplace les $p_{\beta_{1}} \ldots p_{\beta_{r}}$ par les $p_{\beta_{1}} \ldots \beta_{r}$.

Université de Paris. 